\title{
Effective potentials and orbits in Weyl conformastatic slender disk
}

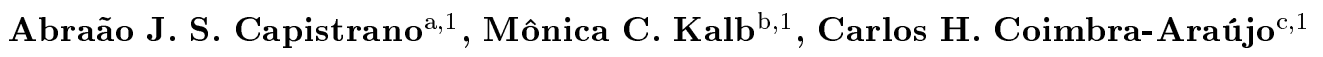 \\ ${ }^{1}$ Universidade Federal do Paraná, 85950-000, Palotina-PR, Brazil. \\ ${ }^{2}$ Applied physics graduation program, UNILA, 85867-670, Foz do Iguaçu-PR, Brazil
}

Keywords: Nearly newtonian limit, gravitation, stars, clusters

\begin{abstract}
By using geodesic equations to obtain a gravitational potential generated from a point-like source, we end up in the concept of a nearly Newtonian gravity to analyse effective potentials of quasi-circular orbits. By means of an approximate solution from an axially static and symmetric Weyl metric, we study an effective gravitational potential to obtain its related rotation curves, orbital planes and orbits. Moreover, using as initial condition a Plummer sphere, some prospects on star cluster disruption are also discussed in this framework.
\end{abstract}

\section{Introduction}

There are an expressive number of exact solutions that have played a very important role in both theoretical and experimental development of General Relativity (GR). For example, one of the most important solutions is the Schwarzschild's, which represents the exterior of a massive body with spherical symmetry. This solution is at the basis of the three classic tests of GR: the displacement of spectral lines due the presence of the gravitational field, the deflection of light passing close to a massive body (stars, galaxies, clusters) and the perihelion precession of planets. Furthermore, this solution gave rise to the concept of a black hole. Solutions representing the gravitational field of a body with axial symmetry play an important role in both Newtonian and GR theory of gravitation, since the natural form of an isolated self-gravitating fluid has axial symmetry. In particular, disk-like configurations are of great interest in astrophysics since they can be used to

a e-mail: capistrano@ufpr.br

be-mail: mckalb@gmail.com

c e-mail: carlos.coimbra@ufpr.br model galaxies, accretion disks and indeed simple axially symmetric stellar systems. In Newtonian gravity, globular clusters and spherical galaxies were calculated by [1] and [2]. [3], [4] and, later, [5] and [6] developed a series of axially symmetric models, described by potential pairs, the associated density functions and also motion equations of the particles in the system, e.g., the effective velocity and rotation curves. More details on the subject can be found in [7] and [8].

In the realm of GR, we use the Weyl axisymmetric metric [9] in cylindric coordinates to obtain a Newtonianlike potential in order to study possible gravitational effects at astrophysical scales. This Newtonian analogue of Weyl metric potential has been extensively studied in literature and can be found, e.g, in gravitation textbooks such as [10, [11] and [12. The so called Weyl metrics define a class of static and axisymmetric solutions to Einstein's equations. The "conformastatic" solution is used to emphasize the restriction of axisymmetric symmetry by using just one "Weyl potential" function $\sigma(r, z)$. This basic idea was applied in previous works to different themes such as apsidal precession in different systems [13, 14, 15, 16] and to the dark matter problem [17.

In this paper, we focus on the obtainment of effective gravitational potentials to study their related orbits as an application of the geodesic slow motion concept in GR to motion of stars in axisymmetric astrophyical configuration. As a toy model, we use galpy ${ }^{1}$ numerical code to study and evaluate how such potentials may respond and reproduce astrophysical systems. In section 2, we discuss the slow motion limit of GR, which simply means that we use only the geodesic equations alone, leaving the deviation equation intact [13, 14, 18,

${ }^{1}$ Available at https ://github.com/jobovy/galpy 
to obtain a nearly-Newtonian limit [19. In section 3, we present the application of the nearly-Newtonian potential resulting from Weyl's metric to the obtainment of rotation curves and orbit of stars. In section 4 , some prospects on N-body simulation of disrupt clusters are commented. In the conclusion section, we present the final remarks.

\section{The slender disk condition}

Starting from the standard RG configuration, a nearlyNewtonian limit can be obtained that carries non-linear effects as an heritage from a relativistic system not under the a priori assumption of a weak gravitational field. This apparent discrepancy between motion and field principles was a real problem in the early days of GR, and it was only enlightened by [18] decades later showing that the Einstein field equations implicitly contained motion equations. In such a process, we investigate the fact that different parameters may lead to different physical configurations, which means that some situations may be obtained by the analysis of geodesic motion and the tuning of the strength of local gravitational filed. For instance, near black holes the stars motion indicate that the velocities of nearby stars are of the order of a few hundreds of kilometers per second which should be describe by a slow geodesic motion in the presence of strong gravitational fields with highestenergy cosmic ray jets and active galactic nuclei [20,21]. In addition, the linear gravitational wave equation and the Schwarzschild weak fields with the parameter $1 / r$ are not velocity-related. It is also important to point out that the parametric post-Newtonian (PPN) expansion is not uniformly valid for large distances [22] and has limitations on the study, for instance, of the dynamics of pulsars 23 , which breaks down in the radiation zone where gravitational waves propagate and boundary conditions are commonly defined. The beforementioned examples give us a window of opportunities to search models or methodologies under those circumstances blending the subtleties of motion and field of GR to comply with astrophysical systems.

In the present model, we start with an idea using a free test particle star that orbits the galaxy-bulge regarded as a point-like source centered at the circular basis of a cylinder by means of Weyl's line element [9]

$d s^{2}=e^{2(\lambda-\sigma)} d r^{2}+r^{2} e^{-2 \sigma} d \theta^{2}+e^{2(\lambda-\sigma)} d z^{2}-e^{2 \sigma} d t^{2}$,

where $\lambda=\lambda(r, z)$ and $\sigma=\sigma(r, z)$ are the Weyl's coefficients. Thus, the exterior gravitational field is given by Einstein's vacuum equations

$$
-\lambda_{, r}+r \sigma_{, r}^{2}-r \sigma_{, z}^{2}=0
$$

$$
\begin{aligned}
& \sigma_{, r}+r \sigma_{, r r}+r \sigma_{, z z}=0, \\
& 2 r \sigma_{, r} \sigma_{, z}=\lambda_{, z} .
\end{aligned}
$$

where the terms $(, r),(, z)$ and $(, r r),(, z z)$ denote respectively the first and the second derivatives with respect to the variables $r$ and $z$. It is important to point out that Weyl's metric does not lose its asymptotes when reduced to Schwarzschild symmetry [9, 24,25] and is also asymptotically flat $[9,12,24,25,26]$. These two features turn such metric an interesting case for application to astrophysical systems.

Under specific conditions, the resulting gravitational field will be different like that of the one produced by Schwarzschild's geometry to avoid Cartan's equivalence 2 [12,27,28. Hence, the diffeomorphism invariance of GR must be broken down by the slender disk condition, i.e., the cylinder thickness $h_{0}$ is much smaller than its radius $R_{0}$, i.e., $h_{0}<<R_{0}$. At first, with this procedure, we avoid to attribute any surface density of the cylinder, which eventually would lead to an singularity in $z$-coordinate like those works of [29, [30] and [31. Since we are interested in the particle's orbit itself, we expand the coefficients $\lambda(r, z)$ and $\sigma(r, z)$ into a Taylor's series in such a way

$$
\begin{aligned}
& \sigma(r, z) \approx \sigma(r, 0)+\left.z \frac{\partial \sigma(r, z)}{\partial z}\right|_{z=0}+\left.z^{2} \frac{\partial^{2} \sigma(r, z)}{\partial z^{2}}\right|_{z=0}+\cdots,(5) \\
& \lambda(r, z) \approx \lambda(r, 0)+\left.z \frac{\partial \lambda(r, z)}{\partial z}\right|_{z=0}+\left.z^{2} \frac{\partial^{2} \lambda(r, z)}{\partial z^{2}}\right|_{z=0}+\cdots
\end{aligned}
$$

Truncating the approximation up to the second order to guarantee the non-linearity in the expansion, we write

$\sigma(r, z)=A(r)+a(r) z+c(r) z^{2}$,

where we denote $A(r)=\sigma(r, 0), a(r)=\left.\frac{\partial \sigma(r, z)}{\partial z}\right|_{z=0}$ and $c(r)=\left.\frac{\partial^{2} \sigma(r, z)}{\partial z^{2}}\right|_{z=0}$.

In addition, we use the same procedure as in eq. (7) to the coefficient $\lambda(r, z)$ and define

$\lambda(r, z)=B(r)+b(r) z+d(r) z^{2}$,

where we denote $B(r)=\lambda(r, 0), b(r)=\left.\frac{\partial \lambda(r, z)}{\partial z}\right|_{z=0}$ and $d(r)=\left.\frac{\partial^{2} \lambda(r, z)}{\partial z^{2}}\right|_{z=0}$. Thus, replacing the coefficients given by eqs. (7) and (8) in the system given by eqs.(2), (3) and (4), one obtains the final form of the coefficient $\sigma(r, z)$

$\sigma(r, z)=\frac{k_{0}}{2} \ln (r)-\frac{c_{0} r^{2}}{2}+a_{0} z+c_{0} z^{2}+c_{1}$,

${ }^{2}$ The Riemann tensors and their covariant derivatives up to the seventh order must be equal. 
and also a closed form for the coefficient $\lambda(r, z)$ given by

$$
\begin{array}{r}
\lambda(r, z)=\frac{k_{0}^{2}}{4} \ln (r)-k_{0} c_{0} \frac{r^{2}}{2}+\frac{1}{4} c_{0}^{2} r^{4} \\
-\left(a_{0}+2 c_{0} z\right)^{2} \frac{r^{2}}{2}+k_{0} a_{0} z+k_{0} c_{0} z^{2}+d_{1},
\end{array}
$$

where $c_{1}$ and $d_{1}$ are true integration constants. As shown in [13, for superior orders, the terms in the coefficients $\sigma(r, z)$ and $\lambda(r, z)$ turn to be redundant. It was found that the initial functions $a(r), c(r)$ can be reduced to constants $a_{0}$ and $c_{0}$, respectively, and $k_{0}$ is a constant factor. The functions $b(r)$ and $d(r)$ are given by

$$
\begin{gathered}
b(r)=k_{0} a_{0}-2 a_{0} c_{0} r^{2} \\
d(r)=k_{0} c_{0}-2 c_{0}^{2} r^{2}
\end{gathered}
$$

In this paper, we focus on the discontentment of simpler solutions and we consider the conformastatic case, i.e., when $\lambda(r, z)=0$, since such coefficient does not directly contribute for the resulting gravitational potential.

\section{The effective potentials and orbits}

In the following, we essentially reproduce the standard results of Misner, Thorne and Wheeler's book [19. In the geodesic analysis of motion, a small deviation from Minkowski's metric does not necessarily depend on the velocity of an arbitrary particle. On the other hand, if once stabilises slow motion $v<<c$, Newtonian coordinates can be used with $x^{4}=t, t$ being the Newtonian time, so that the spatial components of the geodesic equation become

$\frac{d^{2} x^{i}}{d t^{2}}=-\Gamma_{\mu \nu}^{i} \frac{d x^{\mu}}{d t} \frac{d x^{\nu}}{d t}-2 \Gamma_{\mu 4}^{i} \frac{d x^{\mu}}{d t}=-\Gamma_{44}^{i}=\frac{1}{2} \delta h_{44, i}$,

where the Latin indices run from 1 to 3 . An arbitrary gravitational scalar field $\Phi$ can be defined as

$\frac{d^{2} x^{i}}{d t^{2}}=-\frac{\partial \Phi}{\partial x^{i}}$

By the comparison between eqs. (13) and (14), one obtains the field $\Phi$ :

$\frac{\partial \Phi}{\partial x^{i}}=-\frac{1}{2} \delta_{i j} \frac{\partial \delta h_{44}}{\partial x_{j}}$,

where $\delta_{i j}$ is the Kronecker delta. It is important to note that the potential $\Phi$ is not necessarily the Newtonian gravitational field because the gravitational field was not a priori assumed to be weak. Moreover, assuming there is not external force, the gravitational field from the source continuously pull the test particle building up by small increments of the metric as

$$
g_{\mu \nu} \approx \eta_{\mu \nu}+\delta h_{\mu \nu}+\left(\delta h_{\mu \nu}\right)^{2}+\cdots
$$

Thus, the set of increments $\delta h_{\mu \nu}$ continuously increases up to a finite value $h_{\mu \nu}$, and eq. 15 can be readily integrated along the geodesic path, and one obtain the nearly-Newtonian potential [19]:

$\Phi_{n N}=-\frac{c^{2}}{2} \int_{0}^{h_{44}} d\left(\delta h_{44}\right)=-\frac{c^{2}}{2}\left(1+g_{44}\right)$.

As seen before, except at the beginning of the free fall, the weakness of a Newtonian gravitational field was not a priori condition. As a result, the component $g_{44}$ of the metric is obtained from an exact solution of Einstein's equations, whose solutions count with the contribution of all metric components. The symmetry group of this equation is similar to the generalized Galilean group. In this case, the Newtonian potential is replaced by eq. (16), which means that due to diffeomorphic transformations are not allowed, and the metric coordinates must be consistent with the metric symmetry of the local gravitational field. It is interesting to note that not only the slow motion condition in static gravitational configurations appears to be applied, but also a particular reduction on the nonlinearity contained in the affine connection. In addition, it may be possible the development of astrophysical models where the metric tensor is not trivial and restricted eventually to be symmetric.

In reality, the motion of stars in spiral galaxies tell that we need a gravitational model capable of describing a slow motion also valid for gravitational fields of any strength. In order to test this nearly Newtonian limit, using eqs. (9) and (16), one can calculate in the galactic plane the related gravitational potential generated by a point-like mass at a distance $r$ given by

$\Phi_{n N}(r, z)=-\frac{c^{2}}{2}\left(1-a_{1} r^{k_{0}} e^{-c_{0} r^{2}+2\left(a_{0} z+c_{0} z^{2}\right)}\right)$.

For convenience, we refer $\Phi_{n N}$ just as Weyl potential.

\subsection{Effective velocity and rotation curves}

In a previous publication [13], it was argued that at Solar system scale the parameter $c_{0}<<1$, which means that the quadratic radial term at eq. (9) evolves slowly as the radial distance increases. At galactic scale, it may be interesting to explore some gravitational effect like that of the rotation curves problem [17. As an example, one obtains a rotation curve by means of an effective radial velocity $v_{\text {eff }}$. Since the slow motion geodesic equation is not invariant under diffeomorphisms we may consider some gravitational stages separately in such a way

$v_{e f f}^{2}=v_{N}^{2}+v_{n N}^{2}$,

which the terms $v_{N}$ and $v_{n N}$ are the Newtonian velocity and the nearly-Newtonian velocities, respectively. 
Due to the slender disk condition, its not possible to simulate the galaxy bulge per se and the passage of a pointlike source to an extended particle distribution is a must. Once much of galactic systems are fairly nonspherical, we expect to reproduce approximated spherical bulges and flat disks. In order to test this hypothesis, we model a mass distribution to extended source in a linearized framework with a Newtonian mass $M(r)$, and we resort to additional potentials to provide a Newtonian velocity $v_{N}$. To our numerical study, we use the Miyamoto-Nagai potential and the Milky-Way-like potential, dubbed as MWpotential as provided in [32]. The Miyamoto-Naygai model 55 provides a thickened disk as an extended form of a Kuzmin disk 4. The resulting potential is given by

$\Phi(r, z)=-\frac{G M}{r^{2}+\left(a^{2}+\sqrt{z^{2}+b^{2}}\right)^{2}}$,

when $a \rightarrow 0$ the potential is reduced to a Plummer sphere [1] and $b \rightarrow 0$ to a Kuzmin disk. To enhance our analysis, we also use a Milky Way's gravitational potential MWpotential with no consideration of the central supermassive black hole's gravity. It is important to note that we use MWpotential as a Milky-Way-like potential in galpy but the code itself calls MWpotential as MWPotential2014. From eq. 17) and using the standard Newtonian formula for calculating the circular equatorial velocity $v(r)=\sqrt{\left.r \frac{\partial \Phi}{\partial r}\right\rfloor_{z=0}}$, the nearly-Newtonian velocity is written as

$v_{n N}(r)=\sqrt{\frac{G M(r)}{2 R_{0}} A(r)}$,

where $R_{0}$ is the optical disk length scale, $M(r)$ is the mass model to be adopted. Moreover, $A(r)$ denotes the term $\left(k_{0}+2 c_{0}(r+1)^{2}\right) a_{1}(r+1)^{k_{0}} e^{-c_{0} r^{2}}$. Accordingly, eq. 20 evinces some important requirement of the effective velocity that vanishes asymptotically. From the theoretical point of view, $v_{n N}$ vanishes in an infinite radius for an asymptotically flat rotation curve. In practice, this means that at maximum finite radius according to observations, the influence of a gravitational pull of the galaxy ceases to be. In addition, we point out that eq.20 does not reach the Newtonian limit. Actually, this situation was expected to happen since the Newtonian limit should be reached at $k_{0}=1, c_{0}=0$, but the radial distance dependence remains in the $A(r)$ term. When one sets $k_{0}, c_{0}=0$, eq. (20) is zero since the Weyl potential $\sigma(r, z)$ vanishes. This result is as a relic of the breakage of diffeomorphic transformations and imposes a constraint on the parameter $k_{0} \neq 0$.

In Fig.(1), we present an idealised rotation curves in the sense that galaxy rotation and inclination effects are not taken into account and shows a necessary asymptotically flat rotation curve as required to analyse the

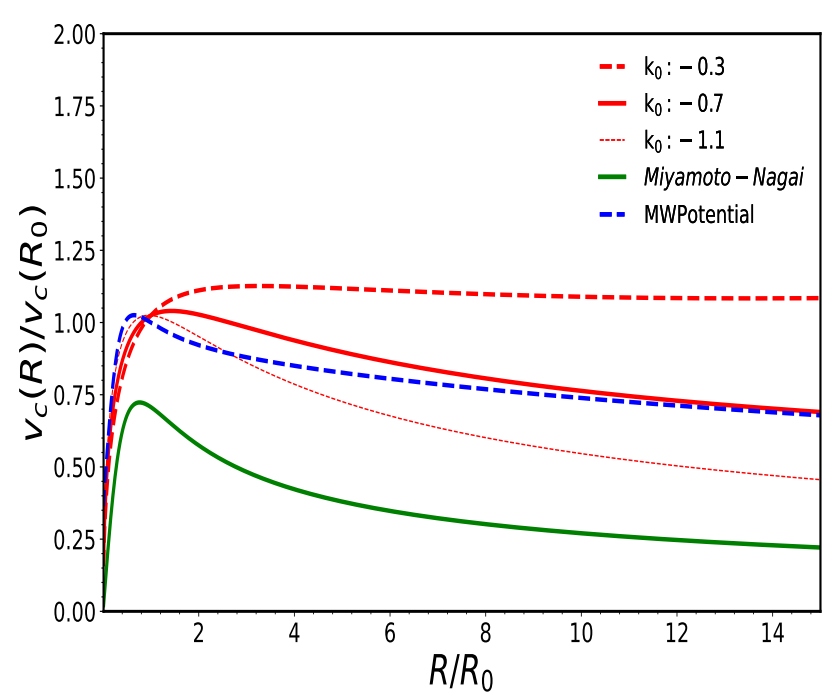

Fig. 1 Rotation curves comparison between the effective velocity with the additional nearly-Newtonian contribution (referred to colour in electric version as the dashed, thick and dotted red lines in the curves). The Miyamoto-Nagai and MWpotential potentials are indicated by the thick green and dashed-thick blue lines. We use galpy units normalized as the velocity $v=1$ at $r=1$.

rotation curve problem. References to colour, the reader is referred to check the electronic version of this article. Rotation curves comparison between the effective velocity with the additional nearly-Newtonian contribution dashed, thick and dotted red lines in the curves. The Miyamoto-Nagai potential and Milk way potential MWpotential are indicated by the thick green and dashed-thick blue lines. The curve of the MiyamotoNagai potential alone is obtained with the parameters $(a, b)=(0.9,0.09)$, while MWpotential rotation curve is obtained by the default parameters defined by galpy code. The complete description of the parameters and properties of MWpotential can be found in table 1 of [32. In the same reference, one finds that galpy assumes by default internal natural units. To implement further calculations, we maintain the standard galpy units, which normalize the resulting velocities set up to value $v=1$ at radius $r=1$. To physical units, it corresponds to a circular velocity of $V=220 \mathrm{~km} / \mathrm{s}$ at $X=8 \mathrm{kpc}$, and velocities and distances are scaled as $v=V /[220 \mathrm{~km} / \mathrm{s}] x=X /[8 k p c]$, respectively. The capital letters $V$ and $X$ correspond to physical velocity and galactic distance.

Fig.(1) shows the curves of the effective velocity (red curves), which are obtained with the composition of Miyamoto-Nagai potential with $(a, b)=(0.9,0.09)$ and three cases are shown for different values of the parameters. The dashed, thick and thin red lines are obtained 
with the values of $k_{0}$ as $(-0.3,-0.07,-1.1)$, respectively. Accordingly, the other parameters $\left(a_{0}, a_{1}, c_{0}\right)$ are set up with the values $(-0.01,-0.01,0.0001)$. We compare the effective curves from the composition of MiyamotoNagai potential and Weyl potential to MWpotential. As shown by the solid thick green line behaviour, the curve resulted from Miyamoto-Nagai potential alone is the lowest. Meanwhile, having MWpotential as a reference, it is possible to obtain curves by varying the parameters of composed potentials with higher (dashed red line) or lower (dotted red line) curves. A duly appropriate curve is shown by the solid thick red line that present a substantial higher rotation curve in the asymptotic regions as compared with MWpotential for a central MiyamotoNagai potential with $(a, b)=(1,0.004)$ which provides a more spherical bulge. This is fairly interesting since it reaches an approximate velocity in physical units as $V \sim 150 \mathrm{~km} . s^{-1}$ at $r \sim 100 \mathrm{kpc}$ that matches the constructed Milky way rotation curve as in [33], which corresponds to in galpy units as $v \sim 0.68$ and $x \sim 12.5$. At first, we also notice that the effect of the inclusion of the Weyl potential improves the rotation curve of Miyamoto-Nagai potential with a higher bulge distribution and a far reaching disk dark component as a result from RG nonlinearity heritage in detriment of a dark matter component. On the other hand, as observed in real rotation curves, the localised peaks and dips are not determined, and they are out of the scope of our this work, which may be reconsidered in a future larger analysis. For this particular case, negative values of the parameters $\left(k_{0}, a_{0}, a_{1}\right)$ are preferred, while $c_{0}$ is close to zero.

\subsection{Star orbits and cluster disruption}

In order to get more understanding of the parameters behaviour, we analyse the relative orbits of stars. This is an important issue for testing our methodology since the different types of orbit stars can tell us about the evolution of galaxies and clusters. In Fig.(2), we present the resulting orbits for possible parameter values as shown in table (1).

For all cases, we fixed the values of parameters in Miyamoto-Nagai potential as $(a=0.9, b=0.09)$. Thus, we obtain the forms of the orbits and reveal related shapes for specific parameter values. In each case, if one varies the values as shown in table (1), it provides small differences in overall shapes. For instance, high values in panel $(a-b)$ in Fig.(2) lead to the increase of trajectories in the $x-y$ planes and will eventually fulfill its whole surface. In panel $(c-d)$, it presents the higher possible values of parameters $k_{0}$ and $a_{1}$. The rosette pattern mimics, e.g., the orbit of NGC 6544 [34]
Table 1 The defined values of parameters $\left(a_{0}, a_{1}, c_{0}, k_{0}\right)$ used in galpy code.

\begin{tabular}{lcccr}
\hline Parameters & $a_{0}$ & $a_{1}$ & $c_{0}$ & $k_{0}$ \\
\hline \hline panel (a-b) & 0 & 0.1 & 0 & -1 \\
panel (c-d) & -0.02 & 1.5 & 0.0001 & 5.5 \\
panel (e-f) & -0.1 & -0.1 & -0.0001 & -3 \\
panel (g-h) & 0.1 & 0.1 & -0.0001 & 3 \\
panel (i-j) & $\rightarrow 0$ & $\rightarrow 0$ & 0 & $\neq 0$ \\
panel (l-m) & 0 & 0.1 & 0 & $>0$ \\
\hline
\end{tabular}

Table 2 The adopted parameters in N-body simulation.

\begin{tabular}{lccc}
\hline Models & $N$ & $M_{c}$ & $R_{c}$ \\
\hline Weyl & 1000 & 1.5 & 1 \\
Weyl + MW (1) & 10000 & 1500 & 1 \\
Weyl + MW (2) & 10000 & 3000 & 100 \\
\hline
\end{tabular}

at $x-y$ plane and/or also indicates a black hole in the center which makes the stars speed-up. We also verified that for values of $a_{0}>0$ compromise the stability of the plane $r-z$ showing random spikes in the resulting orbit or even a crash in galpy orbit integration. For smoother orbits, we then define $a_{0} \leq 0$. For the panel $g-h, a_{1}$ must be small for positive $k_{0}$. In most cases, if we reverse the signs of $\left(a_{0}, a_{1}\right)$, they will not change the overall shape of the curves.

For the combination of MWpotential with Weyl potential, we found that two possible cases happen. The first one happens when $k_{0}<0$ for null values of $\left(a_{0}, c_{0}\right)$ and small $a_{1}$ that provides a rosette orbit. The second case is for $k_{0}>0$, and keeping the same previous values of the parameters, one obtains a tube shape in the $x-y$ plane while in the $r-z$ plane, one reaches an edge-on orbit, which eventually might lead to chaotic orbit. As an overall result, we obtained basically box and loop orbits, which they appear to be compatible with disk-like and elliptical galaxies.

\section{Disrupting clusters}

In the following, we make a first toy model of N-body simulation of clusters using the beforementioned combined potentials. In other to produce stable simulations, we use galpy code and amuse ${ }^{3}$ module [35, 36, 37, 38, 39 provided by the following assumptions: the cluster is in dynamical equilibrium, Boltzmann's molecular chaos is required (test stars and related fields are fairly independent). The total mass $M_{i}$ of the cluster of

3 https://doi.org/10.5281/zenodo.4946130 

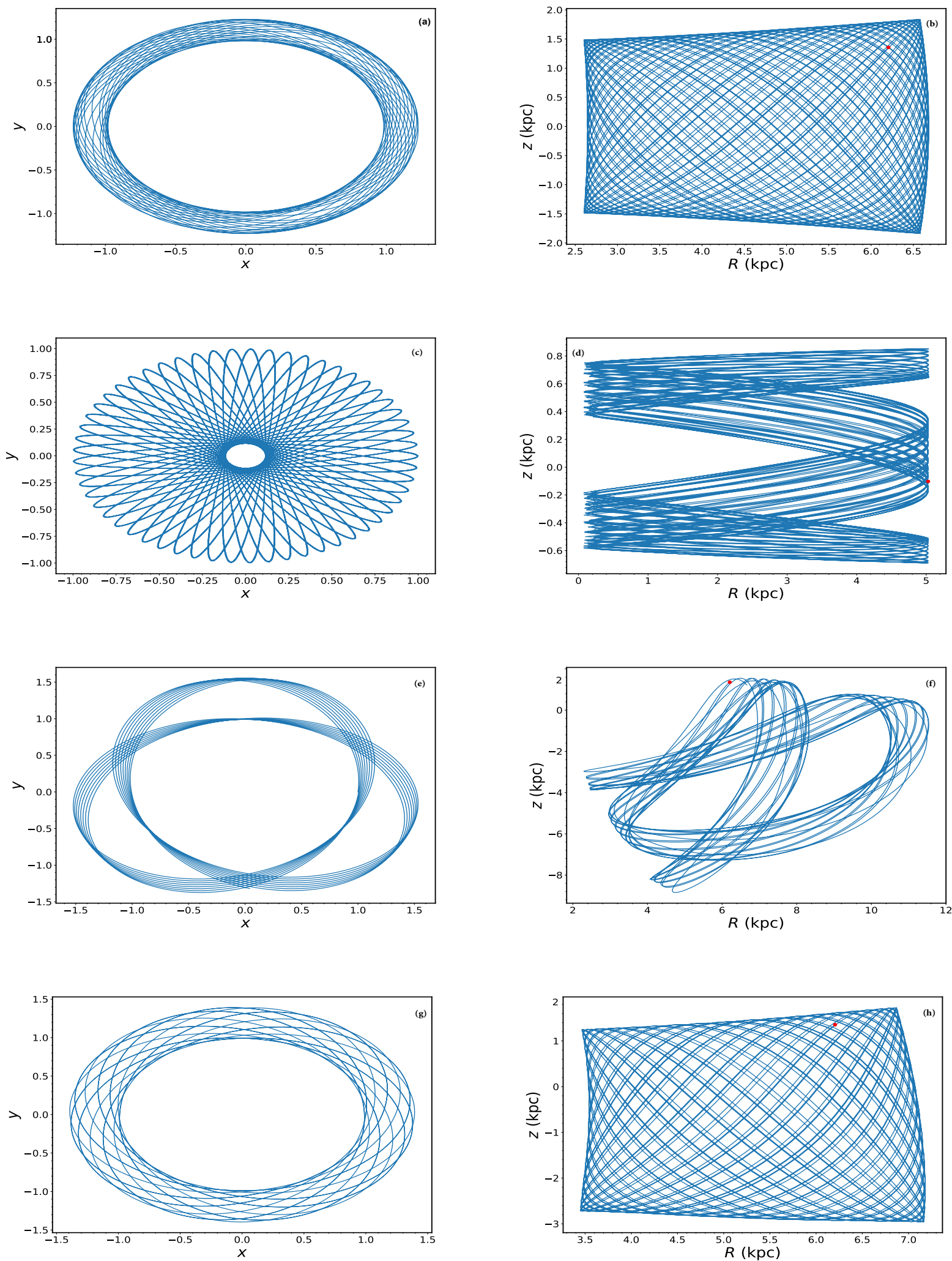

Fig. 2 Orbit collection of different types of orbit in axial symmetric galaxy model for different possible values of the parameters. The panels refer to the composition of Miyamoto-Nagai potential and Weyl potential. The red dot signs the globular cluster Omega Centauri's orbit. 

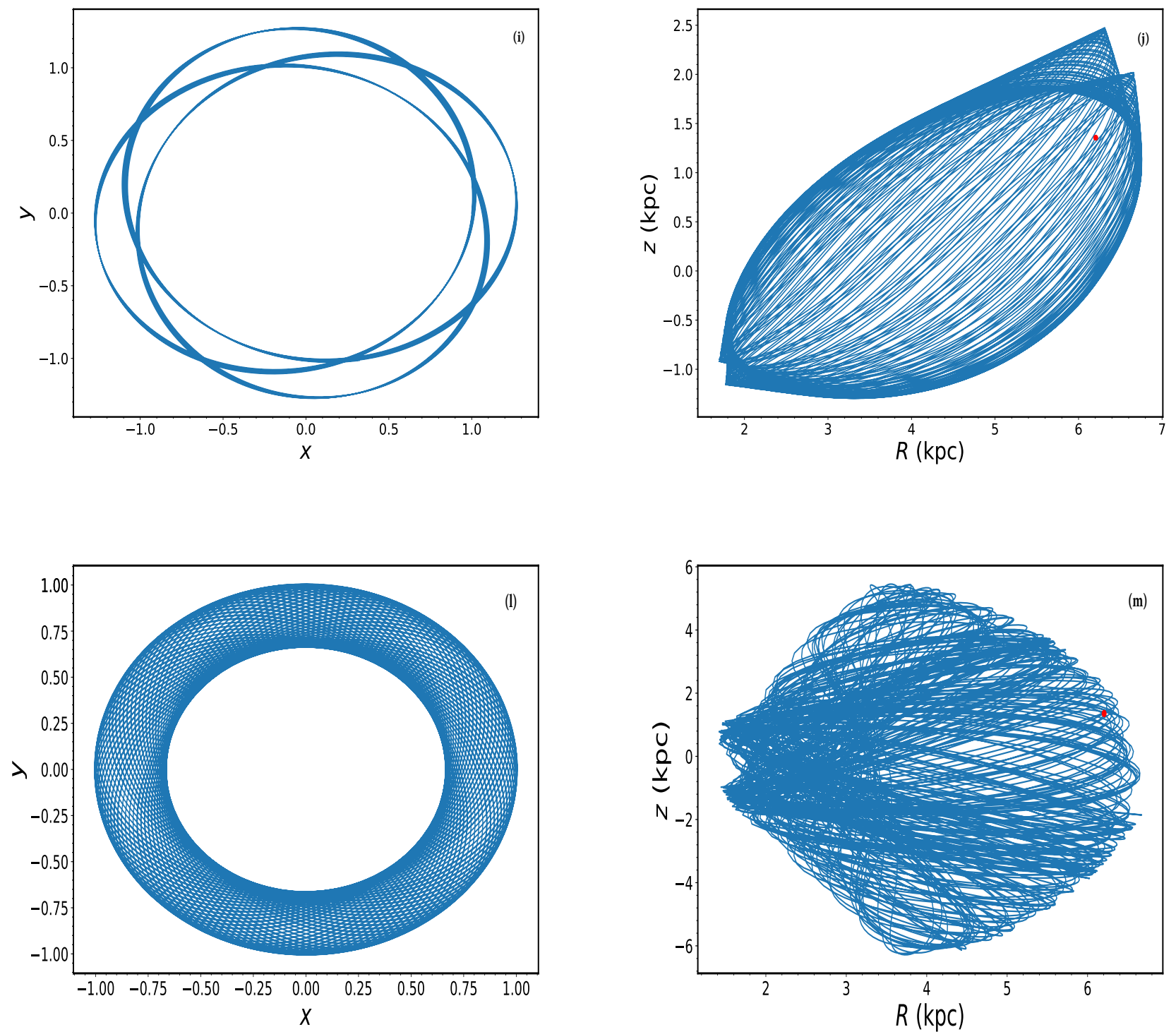

Fig. 3 Orbit collection of different types of orbit in axial symmetric galaxy model for different possible values of the parameters. The upper and middle panels refers to the composition of Mwpotential and Weyl potential. The red dot signs the globular cluster Omega Centauri's orbit.

set of $i$-stars is defined as $M_{i}=\sum_{j=1}^{i}$ as a Plummersphere cluster and an additional external potential must be added to evaluate the evolution of the system with time. For our simulation, we set Weyl potential with the values of the parameters set as $\left(a_{0}, a_{1}, c_{0}, k_{0}\right)=$ $(-0.01,-0.01,0.0001,-0.5)$. For the initial cluster parameters, we have that $N$ accounts for the number of stars, $M_{c}$ is the cluster mass in sun mass units and cluster radius $R_{c}$ in parsec units. In table (2), we present the parameters used in considering Weyl potential alone and in conjunction with MWpotential in two cases varying the cluster mass and the number of particles. We use the initial velocity and radius as default by galpy. As a toy model, we check the Weyl potential in the top panels in Fig.(4). The evolution of the system clearly indicates a fast disruption of a related cluster. When associated with MWpotential in the case (1)(middle panel) the disruption occurs earlier and (2) (bottom panel) occurs later due to more massive stars, but we do not have much differences in evolution with the variation of star masses and the overall aspect of initial phase of disruption where the edge-on stars start falling apart but the cluster center remains unspoil. 

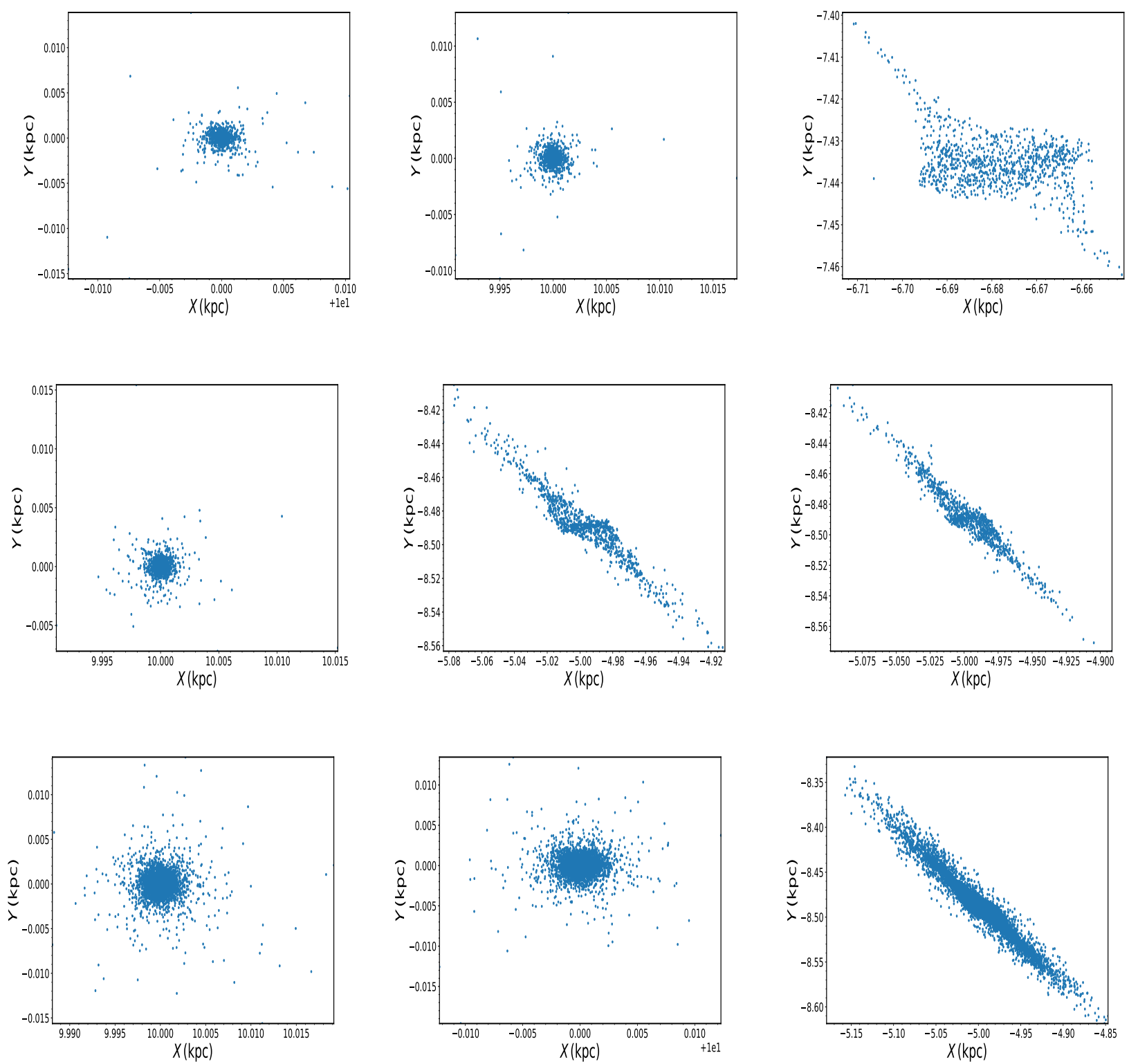

Fig. 4 Numerical simulations of Wey potential alone (top panels) and the conjunction of Weyl potential and MWpotential in middle and bottom panels for different cluster masses and radii (cases (1) and (2), respectively.) in early stages of disruption.

\section{Final remarks}

In this paper, we discussed the slow motion in GR. When considering the slow motion of stars near galaxies, also including near the bulge core, the slow motion breaks down the general covariance of GR but not Einstein's equations which still are valid. In this terms, from using the geodesic equations alone, we are led to "in-between" gravitational potential close to but stronger than Newtonian gravity, the nearly-Newtonian potential dubbed by the traditional Misner, Thorne and Wheeler's book. We recovered such aspect of GR foun- dation in terms of a possible astrophysical implications. As it happens, we proposed a toy model from an axisymmetric Weyl metric under a slender disk condition, which means that the height of the disk can be considered as such smaller than its radius. Thus, we obtained a related gravitational potential used as a toy model in conjunction with Miyamoto-Nagai and MilkyWay-like potentials adopted in galpy python code. We obtained some promising projections when comparing the rotation curves between the conjuncted potential (Miyamoto+Weyl) and Milky-Way-like potential with a steady rotation curve in the galaxy outskirts. It shows 
that the slender Weyl potential adds a considerable contribution to the far reaching dark sector of a galaxy due to nonlinearity propagation resulting from Einstein's equations. Of course, Weyl metric is a symmetric and static metric, and some simplifications were adopted in our analysis since its not possible to include neither rotation nor projection effects. Even so, concerning star orbits, we obtained basically box and loop orbits, which appears to be compatible with disk-like and elliptical galaxies, as expected. This result seems to be generalized somehow in terms of oblate/prolate orbit family should be investigated in a further study using Zipoy-Vorhees family metric. In cluster N-body simulation, we obtained faster disruption cluster evolution adding Weyl potential to Miyamoto-Nagai and MilkyWay-like potentials which serve merit of further applications. As prospects, oblate/prolate metric will be investigated and will be reported elsewhere.

\section{Acknowledgments}

Abraão J. S. Capistrano thanks Fundação Araucária/PR for the Grant CP15/2017-P\&D. Mônica C. Kalb thanks the Coordination for the Improvement of Higher education Personnel Brazilian agency (CAPES) for the scholarship grant.

\section{References}

1. Plummer H. C., 1911, Mon. Not. R. Astron. Soc. 71, 460.

2. King I. R., 1966, A.J., 71, 64.

3. Toomre A., 1963, ApJ, 138, 385.

4. Kuzmin G. G., 1956, AZh, 33, 27.

5. Miyamoto M., Nagai R., 1975, Pub. Astron. Soc. Japan, 27, 533 .

6. Nagai R., Miyamoto M., 1976, Pub. Astron. Soc. Japan, 28, 1.

7. Satoh G., 1980, Pub. Astron. Soc. Japan, 32, 41.

8. Binney S., Tremaine S., 1987, Galactic Dynamics. Princeton Univ. Press, Princeton, NJ.

9. Weyl H., 1917, Ann. Phys., 359, 117.

10. Hartle J.B., 2003, Gravity: An Introduction To Einstein's General Relativity. Princeton Univ. Press, Princeton, NJ.

11. Griffiths J.B., Podolsky J., 2009, Exact Space-Times in Einstein's General Relativity. Princeton Univ. Press, Princeton, NJ.

12. Stephani H., Kramer D., MacCallum M., Hoenselaers C., Herlt E., 2003, Exact Solutions of Einstein's Field Equations. Princeton Univ. Press, Princeton, NJ.

13. Capistrano A.J.S., Roque W.L., Valada R.S., 2014, Mon. Not. R. Astron. Soc., 444, 1639-1646.

14. Capistrano A.J.S., Penagos J.A.M, Alárcon M.S., 2016, Mon. Not. R. Astron. Soc., 463, 1587-1591.

15. Capistrano A.J.S., Seidel P.T.Z., Cabral L.A., 2019, Eur.Phys.Journ. C, 79, 1-8.

16. Capistrano A.J.S., Seidel P.T.Z., Neves V., 2019, Astrophys.Space.Sci., 364, 47.
17. Capistrano A.J.S., Barrocas G.R.G., 2018, Mon. Not. R. Astron. Soc., 475, 2204-2214.

18. Infeld L., Plebanski J., 1960, Motion and Relativity, Pergamon Press.

19. Misner C., Thorne K.S., Wheeler J.A., 1973, Gravitation, W.H. Freeeman \& Co.

20. The Pierre Auger Collaboration, 2007, Science, 318, 938.

21. The Pierre Auger Collaboration, 2017, Science, 357, 6357.

22. Burke W. L., 1969, "The coupling of gravitational radiation to nonrelativistic sources", Dissertation (Ph.D.), California Institute of Technology http://resolver.caltech.edu/ CaltechETD: etd-10152002-090530.

23. Wex N., 2015, "Testing the Motion of Strongly SelfGravitating Bodies with Radio Pulsars", p. 653, In: Fundamental theories of physics, Eds.: D. Puetzfeld, C. Lämmerzahl, B. Schutz, Springer Intern. Publishing.

24. Rosen N., 1949, Rev. Mod. Phys., 21, 503.

25. Zipoy M.D., 1966, Jour. Math. Phys., 7, 1137.

26. Gautreau R., Hoffman R.B., Armenti A., 1972, Il Nuovo Cimento B, 7(1), 71-98.

27. Cartan E., 1927, Ann. Soc. Pol. Mat 6, 1.

28. MacCallum M.A.H., 2006, 28th Spanish Relativity Meeting (ERE05), AIP Conf.Proc. 841, 129-143, gr-qc/0601102.

29. Vogt D., Letelier, P.S., 2008, Mon. Not. R. Astron. Soc., 384, 834-842.

30. Gutiérrez-Piñeres A.C., González G.A., Quevedo H., 2013, Phys. Rev. D, 87, 044010.

31. González G.A., Gutiérrez-Piñeres A.C., Ospina P.A., 2008, Phys. Rev. D, 78, 064058.

32. Bovy J., 2015, Astrophys. J. Supp. 216, 29.

33. Huang Y. et al., 2016, Mon. Not. R. Astron. Soc., 463,3, 2016, 2623-2639.

34. Ramos R.C. et al., 2017, A\&A, 608.

35. Barnes J., Hut P., 1986, Nature, 4, 324.

36. Pelupessy F.I. et al., 2013, A\&A 557, 84.

37. Zwart P.S. et al., 2009, New Astronomy, 14, 4, 369-378.

38. Zwart P.S. et al., 2013, Computer Physics Communications 183, 456-468.

39. Zwart P.S. McMillan S.L.W., 2018, Astrophysical Recipes: the art of AMUSE, AAS IOP Astronomy publishing (411 pp.). 